\title{
Enseñanza de la metodología de la investigación en ciencias sociales: el punto de vista del alumno de máster
}

\author{
Teaching research methods in social sciences: \\ A perspective from master's students
}

\author{
Michela MONTESI ${ }^{1}$ \\ Aurora CUEVAS-CERVERÓ1 \\ María Teresa FERNÁNDEZ-BAJÓN ${ }^{1}$
}

\section{Resumen}

Pretendemos conocer la experiencia del alumnado del Máster en Gestión de la Documentación, Bibliotecas y Archivos de la Universidad Complutense de Madrid en el aprendizaje de la metodología de la investigación. Las áreas exploradas incluyen la percepción del trabajo colaborativo, el papel de las emociones, las implicaciones de las competencias investigativas a nivel profesional, y finalmente el rol del tutor del Trabajo de Fin de Máster. Se realiza una encuesta en la que han participado 26 alumnos de máster inscritos en los cursos 2014-2015 y 2013-2014, tanto en la modalidad virtual como presencial. Los resultados apuntan a la necesidad de equilibrar el trabajo colaborativo con el seguimiento individual del alumno. Ponen de relieve asimismo que un entramado importante de emociones es consustancial a la experiencia de aprendizaje y que la mayoría de éstas son positivas. Es más difícil apreciar las implicaciones de las competencias investigativas para la esfera profesional debido al desconocimiento de los contextos profesionales concretos de los alumnos. Las actividades que los alumnos consideran como más creativas para la investigación suponen la discusión del trabajo propio (tutorías con el tutor) o del trabajo de otros estudiantes (asistencia a defensas de Trabajo de Fin de Máster). Finalmente, la figura del tutor se destaca en cuanto a su importancia en el aprendizaje de la metodología, siendo especialmente relevante su área de investigación.

Palabras clave: Emociones. Enseñanza superior. Metodología de la investigación. Tutor.

\begin{abstract}
The aim of this paper is to understand students' experience of research methodology teaching, through a survey with students enrolled in the Master's Degree Program in Information Management, Libraries and Archives at the Complutense University of Madrid. The analyzed themes included the students' perception of collaborative work, influence of emotions, implications of research competencies at the professional level, and the role of supervisors. Twenty-six master's students, both online and on-site, were surveyed among those enrolled in the academic years 2014-2015 and 2013-2014. Results show the need to foster collaborative work with individual work in a balanced way. Furthermore, emotions, especially positive ones, appear to intertwine heavily with the learning experience. It is more difficult to appreciate the implications of research competencies for the professional sphere because of differences in the professional context of all students involved. The activities that students perceive as more creative include discussions of one's own work (especially with the supervisor) as well as discussions of other students' work (attendance at Master Thesis Defenses). Finally, supervisors stand out as important figure during the learning of research methodology, as their area of expertise is particularly relevant.
\end{abstract}

Keywords: Emotions. Higher education. Research methodology teaching. Supervisor.

\footnotetext{
1 Universidad Complutense de Madrid, Faculdad de Ciencias de la Documentación, Departamento de Biblioteconomia y Documentación. Calle Santísima Trinidad, 37, 28010, Madrid, España. Correspondencia a nombre de/Correspondence to: M. MONTESI. E-mail: <mmontesi@pdi.ucm.es>.
} 


\section{Introdución}

La enseñanza de la metodología de la investigación es un tema emergente en el debate sobre la educación superior, especialmente desde que la formación del alumnado en investigación se ha convertido en un objetivo prioritario para muchas universidades. Prueba de ello son algunas revisiones de la literatura sobre la pedagogía de la metodología de la investigación en las ciencias sociales que se han venido publicando en los últimos años. Entre éstas, Wagner et al. (2008) argumentan que, aunque existen numerosos manuales y recursos para la investigación en distintas disciplinas de las ciencias sociales, en realidad es muy limitada la literatura que ofrece apoyo y referencias para la pedagogía propiamente dicha. Mucha de esta literatura, además, consistiría en experiencias y estrategias docentes que el profesorado emprende muchas veces por iniciativa individual, conocemos aún poco sobre la eficacia de estas estrategias en el aprendizaje del estudiante y sobre cómo los estudiantes realmente aprenden (EARLEY, 2014). Kilburn et al. (2014a) subrayan que la creciente publicación de las iniciativas y estrategias individuales del profesorado no sustituye de manera alguna a la evidencia empírica, todavía prácticamente inexistente, aunque tiene el mérito de estimular el debate sobre la enseñanza de la metodología. Como consecuencia de este debate, los autores aprecian positivamente la naturaleza interdisciplinaria de muchos enfoques pedagógicos, así como el esfuerzo del profesorado para ajustarse a las necesidades concretas del alumnado.

La literatura suele coincidir sobre la relación de alimentación recíproca entre docencia e investigación, aunque para disciplinas como las ciencias de la documentación cabe mencionar una tercera vertiente profesional. Gouldin y Usherwood (2003) consideran que la interrelación entre enseñanza, investigación y práctica es clave para incorporar la investigación en el currículo formativo del Department of Information Studies de la Universidad de Sheffield. Sin embargo, desde el punto de vista del profesorado, este enfoque puede plantear problemas, como una disposición no positiva para la investigación (ALDANA DE BECERRA; JOYA RAMÍREZ, 2011), o la adopción de paradigmas educativos tradicionales por lo menos en algunos contextos como Venezuela (RUIZ BOLÍVAR; TORRES PACHECO, 2005). Incluso en el ámbito anglosajón, no queda claro si las estrategias docentes individuales publicadas en la literatura pueden considerarse ya como una base pedagógica para la enseñanza de la metodología de la investigación en ciencias sociales. El enfoque que parece dominar en muchas experiencias docentes hace hincapié en tres ejes fundamentales, a saber, la implicación del alumno en el proceso de investigación, por ejemplo, a través de experiencias de primera mano en contextos reales, la reflexión crítica, y las actividades prácticas (KILBURN et al., 2014b). Lo que aún queda por conocer es cómo el alumnado concibe, vive y aplica las enseñanzas metodológicas en distintos contextos, incluido el profesional.

El presente estudio pretende contribuir a la literatura existente sobre la enseñanza de la metodología en las ciencias sociales, y lo hace centrándose en el punto de vista del alumno de máster en el área de las ciencias de la documentación. Se basa en un proyecto de innovación docente previo (FERNÁNDEZ-BAJÓN et al., 2015), en el cual, con el objetivo de validar el programa de un curso online de metodología para la realización de Trabajos de Fin de Máster (TFM), se recopiló la opinión de un grupo focal de 6 alumnos del Máster en Gestión de la Documentación, Bibliotecas y Archivos de la Universidad Complutense de Madrid (UCM) sobre su experiencia. El Máster en Gestión de la Documentación, Bibliotecas y Archivos ofrecido por la UCM, en cuanto máster de investigación, incluye en su planificación docente la disciplina Metodología de la investigación científica, obligatoria para todas las especialidades. La asignatura pretende proporcionar un marco teórico y práctico a partir del cual, los estudiantes puedan afrontar de manera real y objetiva las diferentes etapas del proceso de investigación. El presente trabajo ofrece los resultados de una encuesta que tiene como objetivo mejorar nuestro conocimiento sobre la experiencia de aprendizaje de la investigación desde el punto de vista del alumno. En el estudio previo mencionado anteriormente (FERNÁNDEZ-BAJÓN et al., 2015), cuatro aspectos emergieron como especialmente salientes con respecto a la experiencia del alumnado: (1) la importancia de los compañeros de curso; (2) la inevitabilidad de ciertas emociones relacionadas con el trabajo de investigación, como el miedo o la incertidumbre; (3) las implicaciones del trabajo de investigación para la esfera profesional; y finalmente (4) la importancia del director. Los resulta- 
dos apuntaban a que la experiencia de aprendizaje de la investigación se percibe por parte de los alumnos como un 'evento existencial'que repercute más allá del puro contexto formativo para abarcar la esfera profesional y de las relaciones, pudiendo asimismo suscitar emociones contrastantes. En cuanto a éstas últimas, la literatura pedagógica coincide en que una buena gestión de las emociones en el aula repercute en el proceso de aprendizaje en la medida en la que "entiende el aprendizaje como un proceso de cambio constante en la forma de conocer, sentir y actuar del estudiante, superando la concepción tradicional de éste como sujeto pasivo de la enseñanza" (CERDÁ SUÁREZ, 2014, p.15). En el presente estudio, entendemos asimismo que las emociones representan además una manera de conocer al alumnado y consecuentemente de favorecer su interacción con el docente.

\section{Procedimientos metodológicos}

Con estas premisas realizamos una encuesta en el mes de mayo de 2015 entre los alumnos matriculados en el Máster en Gestión de la Documentación, Bibliotecas y Archivos de la UCM en los cursos 20142015 y 2013-2014, tanto en la modalidad presencial como en la virtual. Se diseñó un cuestionario de 26 preguntas, 24 de las cuales son preguntas cerradas y 2 abiertas (UNIVERSIDADE COMPLUTENSE DE MADRID, 2015). Las preguntas abiertas son difíciles de procesar aunque sus aportaciones han resultado valiosas y en nuestro caso la dificultad de proceso ha sido menor pues la muestra ha resultado asequible. Para dar forma a la encuesta se utilizó un formulario de Google Drive que permite responder a través de Internet, aunque los alumnos se contactaron por diversos canales. Parte del alumnado se contactó a través de la Coordinación del Máster (sobre todo los de la modalidad virtual) y a través del campus virtual. También se solicitó la participación de algunos alumnos presencialmente al final de una clase obligatoria el día 25 de mayo de 2015. La participación de los alumnos fue anónima. Los alumnos matriculados en el curso 2013-2014 ascienden a 56 y los del curso 2014-2015 a 45, y las respuestas totales obtenidas fueron 26 , es decir que la tasa de respuesta fue de un $25,8 \%$. La tasa de respuesta relativamente baja puede deberse a que la encuesta se realizó al final del curso académico, siendo cercana la fecha de los exámenes. Específicamente, teniendo en cuenta los datos de la Tabla 1, la tasa de respuesta para los alumnos del curso 2013-2014 fue de un 7,0\%, mientras que para los del curso 2014-2015 fue de un 48,0\%, lo cual indica que la encuesta es mucho más representativa de los alumnos del curso 2014-2015, que todavía asistían a clase cuando se entregó el cuestionario. El $11,0 \%$ de los alumnos encuestados son hombres.

De los demás datos demográficos que permiten caracterizar a la muestra de participantes (Tabla 1) cabe destacar que el 68,0\% de los estudiantes encuestados son de nacionalidad española mientras que el resto comprende alumnos de distinta procedencia, en particular china (19,2\%), mejicana (3,8\%), rumana $(3,8 \%)$ y senegalesa (3,8\%). En cuanto a la situación laboral de los alumnos encuestados, el 52,0\% se encuentra trabajando en la actualidad, y de ellos, el 64,0\% está trabajando o ha trabajado en el ámbito de la documentación. La gran mayoría de los participantes en el momento de cumplimentar la encuesta estaba realizando su Trabajo de Fin de Máster (76,0\%) y había recibido algún tipo de enseñanza sobre metodología (65,0\%).

Tabla 1. Resumen de datos demográficos de los participantes y formación en metodología/estado del Trabajo de Fin de Máster.

\begin{tabular}{lccc}
\hline \multicolumn{4}{c}{ Curso académico } \\
\hline & Alumnos & Respuestas & $\%$ \\
\hline $2013-14$ & 56 & 4 & 7,1 \\
$2014-15$ & 45 & 22 & 48,8 \\
Total & 101 & 26 & 25,8 \\
\hline Nacionalidad de los 26 alumnos que participaron en la encuesta \\
\hline \multicolumn{4}{c}{ Alumnos } \\
\hline Española & encuestados & $\%$ \\
China & 17 & 65,4 \\
Colombiana & 5 & 19,2 \\
Mejicana & 1 & 3,8 \\
Rumana & 1 & 3,8 \\
Senegalesa & 1 & 3,8 \\
\hline Situación laboral & 1 & 3,8 \\
\hline Trabaja & & \\
No trabaja & 14 & 53,8 \\
Otras situaciones & 11 & 42,3 \\
\hline Especialidad profesional & 1 & 3,8 \\
\hline Trabaja o ha trabajado en el ámbito & & \\
de la documentación & & \\
No trabaja ni ha trabajado en el & & \\
ámbito de la documentación & & \\
\hline
\end{tabular}

Fuente: Elaboración propia (2016). 
Los datos obtenidos se han analizado utilizando Excel 2010 (Microsoft Office, Albuquerque, NM, United States) e Statistical Package for Social Sciences (SPSS Inc., Chicago, llinois, United States) versión 22 para la creación de tablas y gráficos. Además de los análisis descriptivos, en algunos casos se ha intentado aislar posibles correlaciones entre las variables, concretamente para ver si las emociones que se dan durante el proceso de investigación para el TFM están relacionadas con factores como el estado del TFM o la formación previa en metodología. Para las pruebas de correlación se utilizó la prueba del Chi cuadrado, que se recomienda en caso de variables nominales y ordinales, como en el presente caso (VAUGHAN, 2001). Es posible apreciar en la sección de resultados, que para muchas variables los participantes expresaron su opinión según unas escalas, dando lugar a datos de tipo ordinal. En prácticamente todos los casos, debido al número reducido de estudiantes participantes, muchas celdas no cumplieron con los valores mínimos de frecuencias necesarios para poder tener en cuenta los valores de significación de la prueba del Chi cuadrado. Para descartar posibles relaciones se tuvieron en cuenta o bien el valor de la prueba exacta de Fisher, en el caso de tablas de contingencia de 2X2, y la Razón de similitud en el caso de tablas contingencias con más de dos filas o columnas (WEINBERG; ABRAMOWITZ, 2008). En algunos casos los datos originarios se editaron, agregando por ejemplo algunos valores en conjuntos más limitados, como sugieren David y Sutton (2004). Por ejemplo, a la hora de establecer las posibles relaciones entre emociones y otras variables, la escala de 4 valores que utilizaron los estudiantes para caracterizar la relación de determinadas emociones con su experiencia de aprendizaje se redujo de 4 (muy relacionado, medianamente relacionado, poco relacionado, nada relacionado) a 2 (relacionado y no relacionado).

\section{Resultados y Discusión}

cuanto al trabajo colaborativo e individual. Entendimos que aquellas competencias que el alumno prefiere desarrollar de manera individual pueden realizarse fuera del aula o, en caso de docencia virtual, pueden prescindir de herramientas de puesta en común con los compañeros. Según podemos observar en el Figura 1, la importancia atribuida por los alumnos al trabajo colaborativo se sitúa en un valor intermedio en una escala de 1 a 5 ( moda=3, media=3,154, mediana=3) y además se aprecia bastante en sintonía con el tipo de docencia impartida en la asignatura ( oda=3, media=3,538, mediana=3). En este sentido, la asignatura parece cumplir con las expectativas de los alumnos en cuanto al trabajo en grupo, confirmando asimismo las conclusiones de Kilburn et al. (2014a) que destacan, en el aprendizaje de la metodología de la investigación, la importancia tanto del trabajo colaborativo como del individual, para poder adaptarse a las necesidades específicas de los alumnos.

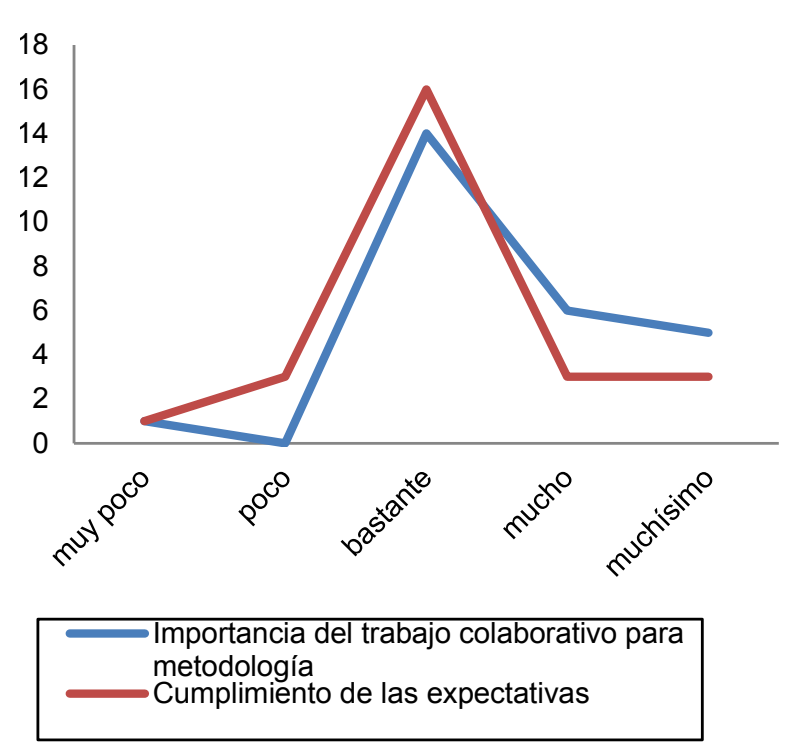

Figura 1. Importancia del trabajo colaborativo y valoración de la asignatura.

Fuente: Elaboración propia (2016).

El Figura 2 representa las preferencias de los alumnos con respecto a cómo desarrollar determinadas competencias propias de la asignatura, si de manera individual o de manera colaborativa. Los participantes constituyeron, de manera significativa, una mezcla de 
trabajo colaborativo e individual, confirmando el resultado anterior de una valoración intermedia del trabajo en grupo. Con referencia a las competencias concretas, se destacan, por un lado, la preferencia por el trabajo individual en cuanto a la búsqueda de información, y por otro la preferencia por el trabajo colaborativo o mixto en la comprensión de conceptos propios de la asignatura (metodología cualitativo versus cuantitativa, variable, muestra), en diferenciar entre distintos tipos de metodología, y en la comprensión de cuestiones éticas. Si es comprensible que a la hora de entender conceptos técnicos o de debatir cuestiones éticas los alumnos manifiesten la necesidad de interactuar con los compañeros, cabe plantearse por qué la búsqueda de información se percibe como una tarea fundamentalmente individual. Los alumnos, en el curso de la asignatura, empiezan a desarrollar su trabajo de fin de máster, cuyo tema es único para cada uno, siendo ésta una posible explicación. Tratándose de una etapa de iniciación a la investigación, los alumnos todavía necesitan desarrollar unos conocimientos de base en el tema del TFM, inclinándose hacia un trabajo individual. Otra posible razón puede encontrarse en la forma de enseñar este tipo de competencias en los estudios de Biblioteconomía y Documentación. A pesar de que la disciplina ha evolucionado, la búsqueda de información se sigue concibiendo todavía como un proceso lineal en el cual tras la detección de determinadas necesidades informativas, se realiza una búsqueda y supuestamente se encuentra la satisfacción de las necesidades de partida. Sin embargo, este proceso es mucho más complejo y puede dar lugar a caminos no lineales y a la puesta en común con otros. Compartir la búsqueda de información con los compañeros de esta manera puede promover la formación de una base de conocimientos en el tema del TFM. La búsqueda de información colaborativa es sin duda alguna un tema en boga en la literatura sobre comportamiento informacional (SHAH, 2014), aunque, repetimos, puede no haberse trasladado a las aulas universitarias.

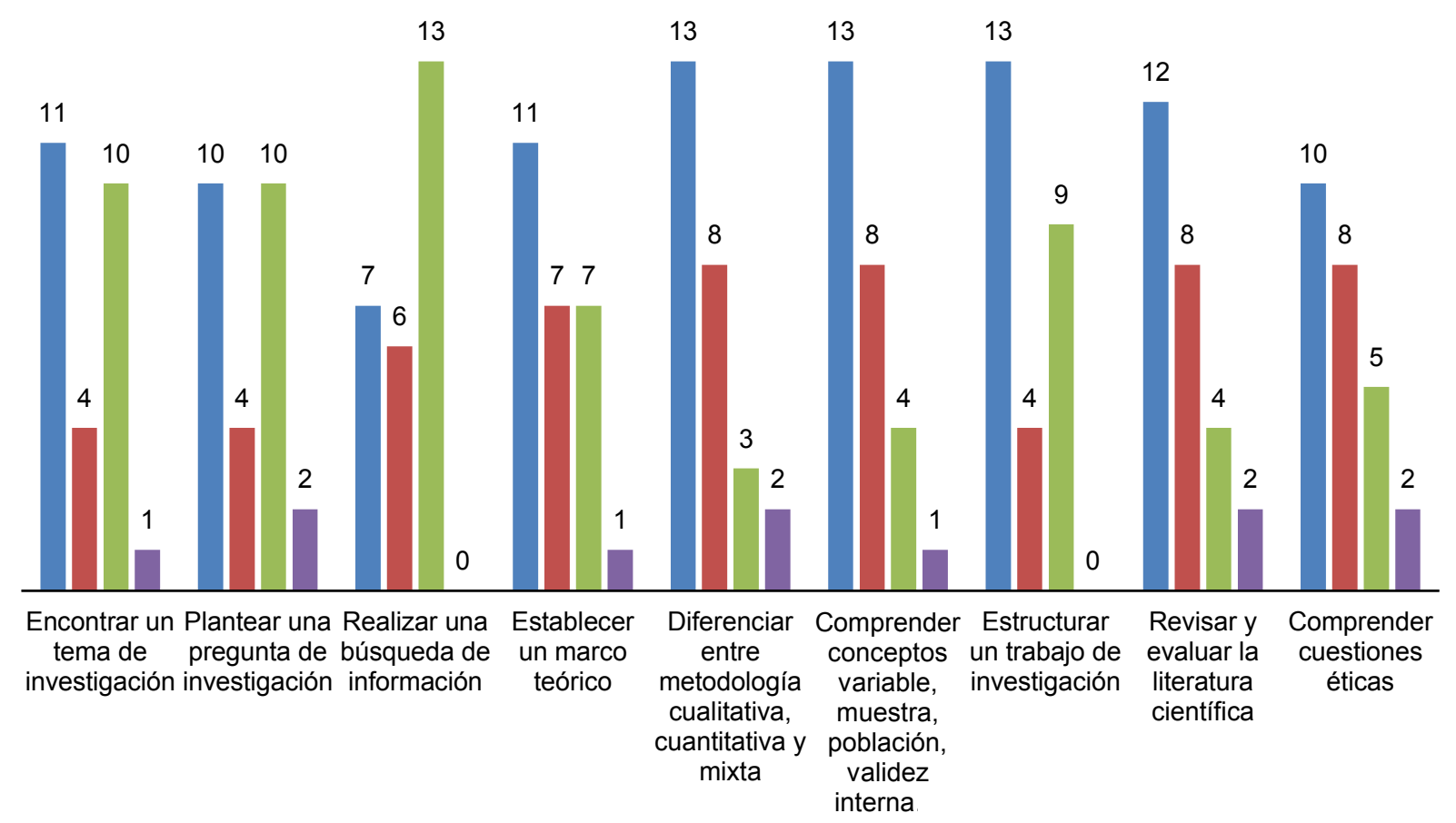

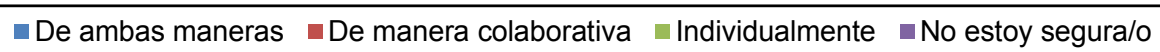

Figura 2. Preferencias en cuanto a tipo de trabajo (colaborativo o individual) por competências. Fuente: Elaboración propia (2016).

El aula no representa el único espacio en el cual los alumnos realizan trabajo colaborativo pues un porcentaje importante de los alumnos declaró trabajar con los compañeros fuera de clase por lo menos a veces (50,0\%). Los participantes declararon compartir su experiencia de estudio en la asignatura y para el TFM con 
otras personas de su entorno (76,9\%), aunque en menor medida con los compañeros de trabajo $(46,2 \%)$. La experiencia de investigación para el TFM parece afectar más a la esfera personal de los alumnos que a la profesional.

\section{Emociones y aprendizaje}

Los participantes se expresaron también con respecto a las emociones relacionadas con su experiencia de aprendizaje en la asignatura y con el TFM. Entre las emociones percibidas más a menudo como muy relacionadas se destacan la preocupación, la satisfacción personal, el entusiasmo y las expectativas de éxito académico, es decir, exceptuando la primera, todas emociones positivas (Figura 3). Los alumnos que comentaron sobre emociones y aprendizaje en la siguiente pregunta abierta se centraron casi exclusivamente en aclarar más bien las emociones negativas pues hablaron sobre todo de miedo, incertidumbre, preocupación, y en menor medida de entusiasmo. La elección del tema de investigación para el TFM, en particular cuando uno procede de una carrera diferente, la elección del tutor, los plazos de ejecución y defensa del TFM son todos factores que pueden desencadenar miedo, preocupación e incertidumbre. En cuanto al tema de investigación, los alumnos pueden experimentar dificultades en la localización de la documentación pertinente (algo que puede tener relación con lo que decíamos antes sobre trabajo colaborativo y búsqueda de información), y dudar sobre la adecuación del mismo a su perfil y personalidad. También pueden dudar en cuanto al método de investigación escogido.

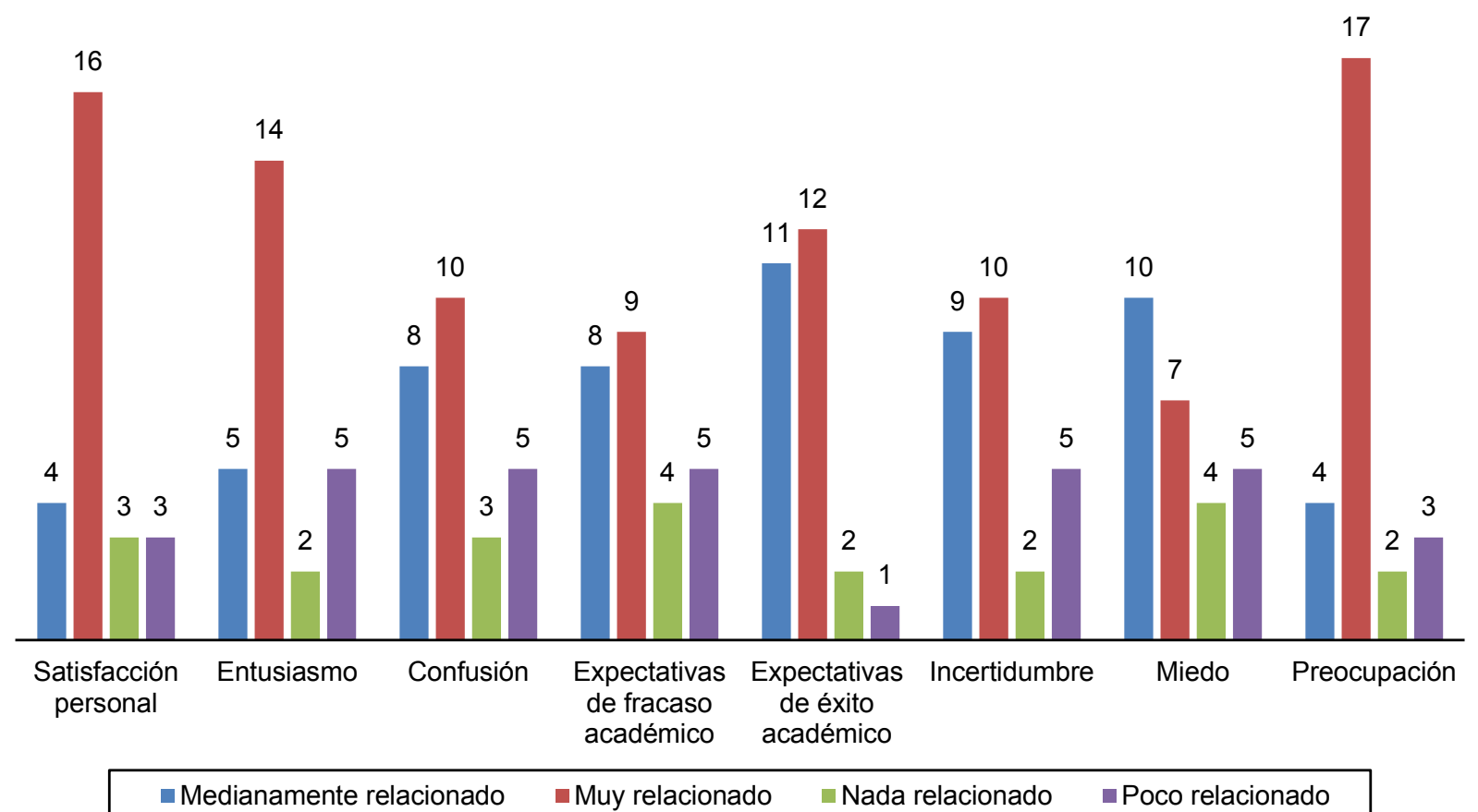

Figura 3. Emociones que intervienen en el aprendizaje.

Fuente: Elaboración propia (2016).

Estas emociones aparecen desde principios de curso y se acentúan especialmente desde que el alumno empieza a trabajar en el TFM convirtiéndose para algunos en 'una presencia constante.' De hecho, los comentarios de los participantes ponen de manifiesto que estas emociones son consustanciales al propio proceso de aprendizaje más que añadidas. En el caso del alumnado virtual, la pérdida de una comunicación fluida con el profesorado puede desencadenar ciertas emociones no necesariamente vinculadas con el TFM, aunque la propia relación con el tutor parece esencial para gestionar estas emociones incluso para alumnos de la modalidad presencial. En los comentarios sobre las emociones positivas, los alumnos mencionaron el entusiasmo y la satisfacción personal más como un deseo o una perspectiva que como reales, puesto que 
se asocian o bien al principio de las clases o a la defensa del TFM y finalización de los estudios (hay que tener en cuenta que en la muestra solo un alumno declaró haber terminado y defendido el TFM).

Para detectar posibles relaciones entre las emociones y otros factores realizamos una prueba de Chi cuadrado entre las variables correspondientes a cada una de las emociones (miedo, incertidumbre, preocupación, expectativas de éxito académico, expectativas de fracaso académico, confusión, entusiasmo) y las variables relativas a: los estudios previos de metodología, el estado del TFM, la situación laboral, el trabajo en el ámbito de la documentación, y los hábitos de comunicación con los compañeros. Las respectivas pruebas de Chi cuadrado, teniendo en cuenta el resultado de la Prueba Exacta de Fisher, descartan la existencia de una relación entre las emociones durante el aprendizaje y los estudios previos de metodología, el estado del TFM y la situación profesional, no pudiendo rechazar la hipótesis nula. En el caso de la relación entre el estado del TFM y la incertidumbre, la preocupación, la confusión y el entusiasmo los valores obtenidos se acercaron más a los niveles de significancia del Test Exacto de Fisher (respectivamente, 0.076, 0.082, 0.068, y 0.069), aunque la relación sigue siendo no significativa. Estos resultados apuntan a que puede tratarse de emociones paralelas y consustanciales al propio aprendizaje en la asignatura de metodología y el TFM.

\section{Actividades relacionadas con la creatividad investigadora}

La creatividad investigadora para los participantes en la encuesta se promueve especialmente en las tutorías con el director del TFM y en la asistencia en defensas de tesis doctorales y TFM (Figura 4), es decir en situaciones en las cuales se debate sobre el trabajo personal propio o de otros pares. Menos influyentes parecen las lecciones impartidas por profesores externos y la asistencia a seminarios y congresos, a pesar de la inversión importante que requieren por parte de las universidades en términos de organización y presupuesto.

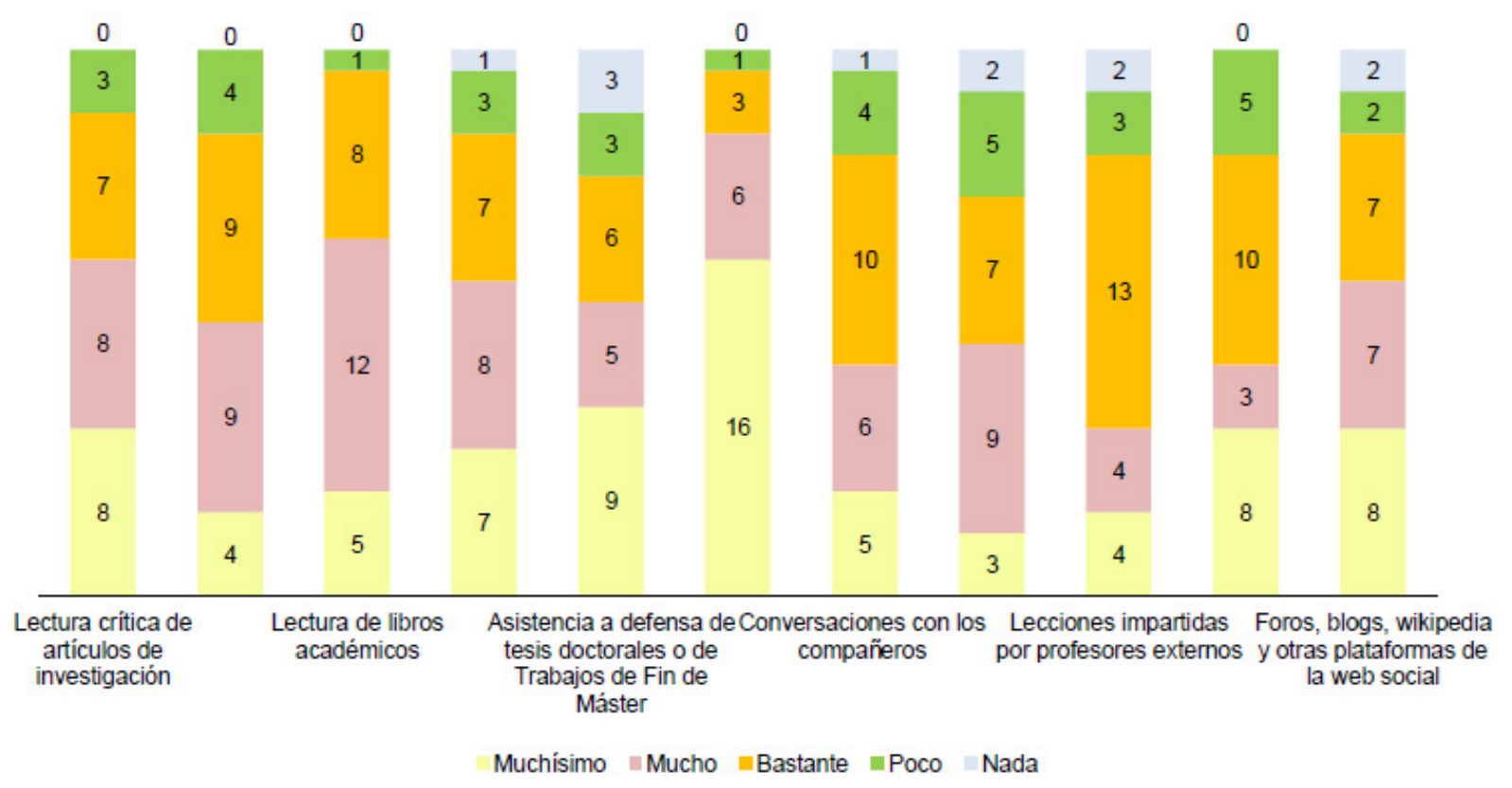

Figura 4. Relación entre actividades y desarrollo de la creatividad investigadora.

Fuente: Elaboración propia (2016). 


\section{Relevancia de la asignatura para el desarrollo de determinados tipos de documentos}

Los alumnos indicaron asimismo su parecer con respecto al papel de la asignatura para el desarrollo de determinadas tareas del ámbito profesional, en concreto la realización de un proyecto, un estudio de mercado, un estudio de usuarios, un informe de resultados, un directorio de recursos especializados, y recursos de apoyo para el aprendizaje. En una escala de cuatro opciones desde 'nada relacionado' hasta 'muy relacionado', la asignatura les parece 'muy relacionada' con la elaboración de un proyecto (para el 34,6\% de los encuestados) y un informe de resultados (para el $34,6 \%$ de los encuestados), y después para un estudio de usuarios (para el 30,7\% de los encuestados) y un directorio de recursos especializados (para el 30,7\% de los encuestados).

\section{Relevancia de la asignatura en la esfera profes- sional}

Los participantes evaluaron la influencia de la asignatura en la esfera profesional puntuando varias competencias desarrolladas en clase en una escala de cuatro opciones desde'no favorece nada'hasta'favorece mucho'. Indicaron que la asignatura 'favorece mucho' en la adquisición de competencias en la búsqueda y evaluación de información (para el 61,5\% de los encuestados) y los métodos y técnicas de investigación (para el 61,5\% de los encuestados), seguidas por la redacción científica (para el 46,1\% de los encuestados) e iniciativa y proposición de proyectos (para el 34,6\% de los encuestados), confirmando los objetivos de la propia asignatura.

\section{El tutor}

Finalmente, los participantes indicaron su parecer con respecto al tutor del TFM. Las razones principales para escoger un tutor tienen que ver con la especialización del mismo, tanto en cuanto a docencia $(15,4 \%)$, como en cuanto a investigación (38,5\%). Secundariamente, están las habilidades de comunicación del propio tutor, siendo razones importantes para escoger al tutor los hechos de que sea accesible $(11,5 \%)$ y que sea fácil comunicarse con él/ella (11,5\%). Sigue la relación con el mismo, pues algunos estudiantes indican que escogieron al tutor por tener una buena relación con él/ella (3,8\%) o haber trabajado previamente con él/ella (11,5\%). Entre los aspectos problemáticos a la hora de escoger a un director, los alumnos, en una pregunta abierta, mencionaron en primer lugar la dificultad de encontrar una persona que reúna una serie de características que el alumno considera positivas, entre otras, según veíamos anteriormente, que tenga conocimientos en la materia de interés, que sea 'de fácil trato' y 'capaz de transmitir las ideas de forma clara y positiva', y que además sea disponible para dirigir el TFM. El hecho de conocer previamente al profesorado es sin duda una ventaja a la hora de aclarar las ideas. Algunos alumnos de la modalidad virtual manifestaron mayores preocupaciones con respecto a este tema, pues para ellos puede ser más difícil desplazarse a la facultad y conocer al profesorado.

Finalmente, los participantes opinaron, en una escala de 1 a 5 (siendo 1 igual a 'muy poco' y 5 igual a 'muchísimo'), sobre las funciones más importantes del tutor. Respecto al rol del tutor, los participantes destacaron su ayuda en la estructuración del trabajo (importantísimo para el 57,6\% de los participantes) y en la elección del tema de investigación trabajo (importantísimo para el 50,0\% de los participantes), así como en la preparación de la defensa del TFM (importantísimo para el 53,8\%). Sorprende que como función más destacada del tutor los alumnos indicaran la ayuda en la estructuración del trabajo, pues disponen de una plantilla específica y reciben formación en la propia asignatura de metodología. Una posible explicación puede ser la escasa familiaridad de muchos alumnos con el texto científico, así como su dependencia del profesor para la adquisición de los contenidos especializados, hecho que desalienta la lectura autónoma. Otra explicación puede ser una distinta implicación emocional con la asignatura y los ejercicios propuestos en clase respecto al propio TFM. Más comprensible es que los alumnos apunten a un importante papel por parte del tutor en la elección del tema de investigación y la defensa del TFM, pues el tutor ofrece conocimientos especializados del área y también del protocolo universitario para la defensa y exposición de trabajos de investigación de los cuales los alumnos aún carecen. 


\section{Conclusión}

Los resultados de este trabajo ponen de relieve varios aspectos y permiten proponer posibles medidas de mejora respecto a la asignatura de Metodología en el ámbito del Máster en Gestión de la Documentación, Bibliotecas y Archivos, y potencialmente para la enseñanza de la metodología en la disciplina. La primera cuestión que investigamos fueron las preferencias de los alumnos con respecto al trabajo colaborativo o individual. Respecto al tipo de trabajo, la asignatura se muestra en sintonía con las necesidades de los alumnos, proponiendo una mezcla de actividades individuales y colaborativas. Los resultados obtenidos, que muestran las preferencias de los alumnos con respecto al tipo de trabajo por competencias, permiten programar mejor las actividades de clase, reservando para el trabajo de casa aquellas tareas que los alumnos prefieren desempeñar individualmente. Un caso aparte en este sentido tiene que ver con la búsqueda de información que los participantes expresaron desarrollar mejor de manera individual que colaborativa. Si todavía en esta etapa de iniciación a la investigación están sentando la base de conocimientos necesaria para realizar su TFM y necesitan por esto dedicación individual, también existen posibles beneficios derivados del trabajo colaborativo en la búsqueda de información que no parecen aprovecharse. En este sentido, cabe proponer una revisión de la forma de enseñar la búsqueda de información en la disciplina para proponerla más como un proceso flexible y colaborativo que preestablecido e individual, independientemente de las preferencias individuales de cada alumno.

Otro resultado que merece la pena subrayar es que tanto la experiencia de aprendizaje de la asignatura como la de investigación para el TFM parecen intrínsecamente relacionadas con un entramado de emociones, positivas y negativas. La preocupación y la satisfacción personal encabezan la lista de las emociones más relacionadas, siendo las causas mayores de preocupación la elección del tutor, la elección del tema de investigación, y el cumplimiento con los plazos de entrega y defensa del TFM. Ni los estudios previos de metodología, ni la situación profesional parecen tener relación con estas emociones que se configuran como más bien consustanciales. En los comentarios de los alumnos tanto de la modalidad virtual como presencial, la relación con el tutor parece esencial para gestionar estas emociones, pues el tutor permite solucionar problemas que suelen desencadenar estas emociones, como por ejemplo la elección del tema de investigación. Si efectivamente, según emerge también de las funciones principales que los participantes asignan al tutor, la elección del tema de investigación se resuelve mejor en colaboración con el tutor, los docentes de metodología deberían trabajar para facilitar la elección del tutor y gestionar los tiempos de ejecución para adecuarse a los plazos establecidos. En este sentido cabe plantear la necesidad de que el trabajo docente sea más colaborativo, contemplando la colaboración más estrecha entre tutores y profesores que imparten la asignatura de metodología. En cuanto a la elección del tutor, muchos alumnos declararon el desconocimiento del profesorado, y sería deseable tanto aumentar la visibilidad del profesorado en el programa de máster como proporcionar información más detallada sobre éste.

En cuanto a las actividades que promueven la creatividad, cabe destacar que las situaciones más creativas para los participantes son aquellas donde se discute su trabajo personal (las tutorías con el director) o el de sus pares (defensas de TFM). Otras actividades formativas, en cambio, como las lecciones de ponentes invitados, muy comunes en los programas de máster, se valoraron de forma menos importante. Quizás la intervención de expertos debería de alguna manera ajustarse a esta exigencia del alumnado de debatir el trabajo propio para poder influir más rotundamente en su trayectoria formativa. Sin embargo, desde el punto de vista del alumnado, el profesorado interno representa el punto más fuerte del programa de máster. Es necesario fomentar su visibilidad, así como apoyar su implicación con el alumnado y adaptación a las necesidades específicas de cada alumno.

En cuanto al rol más prominente del tutor, los alumnos manifestaron la necesidad de su ayuda en la estructuración del TFM, a pesar de recibir una formación específica previa y plantillas destinadas a estructurar el TFM. Las posibles intervenciones de las docentes de Metodología pueden tener en cuenta 
la necesidad de mayor familiaridad con la literatura científica, fomentando la lectura autónoma de textos científicos.

Finalmente, es difícil apreciar las implicaciones de mejores competencias investigativas en el ámbito profesional, sobre todo debido a la gran diversidad de entornos laborales en los cuales pueden estar trabajando los distintos alumnos. Para comprender la repercusión de la formación en investigación para el entorno profesional es probablemente más acertado un enfoque cualitativo que permita primero aislar las posibles variables influyentes. El estudio ha puesto de relieve que la experiencia del TFM es más importante en la esfera personal del alumnado. En el diálogo de las instituciones educativas con alumnos y alumnos potenciales, las posibilidades de desarrollo personal deberían enfatizarse por encima de las posibles salidas profesionales.

\section{Colaboradores}

Todos los autores han contribuido en la concepción y diseño del estudio, análisis de datos y redacción final.

\section{Referencias}

ALDANA DE BECERRA, G. M.; JOYA RAMÍREZ, N. S. Actitudes hacia la investigación científica en docentes de metodología de la investigación. Tabula Rasa, v. 14, p. 295-309, 2011.

CERDÁ SUÁREZ, L. M. Gestión de las emociones en el aula: una experiencia internacional sobre el liderazgo y el desempeño docente. Sistemas, Cibernética e Informática, v. 11, n. 2, p. 14-20, 2014.

DAVID, M., SUTTON, C. D. Social research: The basics. London: Sage, 2004.

EARLEY, M. A. A synthesis of the literature on research methods education. Teaching in Higher Education, v. 19, n. 3, p. 242-253, 2014.

FERNÁNDEZ-BAJÓN, M. T. et al. Proyecto de innovación y mejora de la calidad docente: MOOC: guía para la elaboración de Trabajos Fin de Grado y Trabajos Fin de Master en Ciencias Sociales. Madri: Faculdad de Ciencias de la Documentación, 2015. Disponible en: <http://eprints.ucm.es/28702/1/presentacion_ memoria\%202015_MOOC.pdf>. Acceso em: 21 oct. 2016.

GOULDING, A.; USHERWOOD, B. Research-led teaching in librarianship and information studies. Journal of Librarianship and Information Science, v. 35, n. 3, p. 137-139, 2003.

KILBURN, D.; NIND, M.; WILES, R. Learning as Researchers and Teachers: The development of a pedagogical culture for social science research methods? British Journal of Educational Studies, v. 62, n. 2, p. 191-207, 2014a.

KILBURN, D.; NIND, M.; WILES, R. Short courses in advanced research methods: challenges and opportunities for teaching and learning. United Kingdom: University of Southampton, 2014b. (Project Report). Disponible en: <http://eprints.ncrm. ac.uk/3601/>. Acceso en: 21 oct. 2016.

RUIZ BOLÍVAR, C.; TORRES PACHECO, V. La enseñanza de la investigación en la universidad: el caso de una universidad pública venezolana. Investigación y Postgrado, v. 20, n. 2, p. 13-34, 2005.

$\mathrm{SHAH}, \mathrm{C}$. Collaborative information seeking. Journal of the Association for Information Science and Technology, v. 65, n. 2, p. 215-236, 2014.

VAUGHAN, L. Statistical methods for the information professional: A practical, painless approach to understanding, using, and interpreting statistics. Medford: Information Today, 2001.

WAGNER, C.; GARNER, M.; KAWULICH, B. The state of the art of teaching research methods in the social sciences: Towards a pedagogical culture. Studies in Higher Education, v. 36, n. 1, p. 75-88, 2008.

WEINBERG, S. L., Y ABRAMOWITZ, S. K. Statistics using SPSS: An integrative approach. Cambridge: Cambridge University Press, 2008.

UNIVERSIDADE COMPLUTENSE DE MADRID. Encuste: metodologia de la Investigación. Madrid: UCM, 2015. Disponible en: <https:// docs.google.com/a/ucm.es/forms/d/e/1FAIpQLSdLMMV5 fSh_MOMFGzl6HDffzlKkZX4lojwWdRDpFKgZ9ib8YA/view form>. Acceso en: 21 out. 2016. 